\title{
Business Process Management
}

\author{
DOI 10.1007/s11576-014-0420-8
}

\section{Die Autoren}

Prof. Dr. Jan vom Brocke ( $\bowtie)$ Department of Information Systems University of Liechtenstein Fürst-Franz-Josef-Strasse 21 9490 Vaduz

Liechtenstein jan.vom.brocke@uni.li

Prof. Dr. Lars Mathiassen, Ph.D. Department of Computer Information Systems Georgia State University PO Box 4015 Atlanta, GA 30302-4015 USA Imathiassen@ceprin.org

Prof. Dr. Michael Rosemann Department of Information Systems Queensland University of Technology

Brisbane

Australien

m.rosemann@qut.edu.au

Online publiziert: 2014-06-03

This article is also available in English via http://www.springerlink.com and http://www.bise-journal.org: vom Brocke J, Mathiassen L, Rosemann M (2014) Business Process Management. Bus Inf Syst Eng. doi: 10.1007/ s12599-014-0330-8.

(C) Springer Fachmedien Wiesbaden 2014
Business Process Management (BPM), deutschsprachig auch als Geschäftsprozessmanagement bezeichnet, verknüpft wirtschaftliche und technologische Perspektiven mit dem Ziel, die Prozesse einer Organisation zu verbessern. BPM befasst sich mit der Effektivität und Effizienz einer Organisation und leistet damit einen signifikanten Beitrag zur Gesamtleistung und Wettbewerbsfähigkeit eines Unternehmens. BPM spielt eine wichtige Rolle, um Innovation und Transformation in Organisationen zu gestalten; es geht heute weit über kostenorientierte Prozessverbesserungen (z. B. Lean, Six Sigma) hinaus und unterstützt Manager bei der Erschließung neuer Wertschöpfungspotenziale (z. B. zuverlässige, nachhaltige Prozesse) sowie insgesamt bei der Erhöhung der Leistungsfähigkeit der Organisation (z.B. flexiblere, innovativere Prozesse).

Aus wissenschaftlicher Sicht bringt BPM Forscher aus verschiedenen, sich ergänzenden Disziplinen zusammen. Untersucht werden sowohl technische als auch methodische und kulturelle Aspekte, mit dem Ziel, ein unternehmensweites und prozessspezifisches BPM zu entwickeln. In den fast 25 Jahren seit den grundlegenden Arbeiten von Michael Hammer haben wir enorme Erkenntnisse in den Gebieten Modellierung, Analyse, Ausführung und Kontrolle von Prozessen gewinnen können. Die BPM-Forschung und -Praxis steht aber weiterhin vor einer Vielzahl an Herausforderungen, insbesondere im Hinblick auf die Integration von BPM in bestehende Management- und ITKonzeptionen, die Erschließung neuer Gestaltungsmöglichkeiten im BPM, z.B. durch mobile, soziale oder cloudbasierte Technologien, sowie die Anpassung von BPM an spezifische Anwendungsbereiche (z. B. wissens- und kreativitätsintensive Bereiche).

Das vorliegende Schwerpunktthema präsentiert Ergebnisse neuester internationaler Forschung der faszinierenden Disziplin BPM. Im Auswahlprozess wurden erweiterte und überarbeitete Manuskripte des hochkompetitiven BPM-Tracks der International Conference on Information Systems (ICIS 2013, Mailand) eingeladen sowie Einreichungen aus dem regulären BISE-Pool berücksichtigt. Es ist uns eine große Freude, Ihnen drei Arbeiten aus der Gruppe dieser hochqualitativen Einreichungen zu präsentieren. Die Arbeiten wurden einem mehrstufigen rigorosen Begutachtungsprozess unterzogen und die Artikel veranschaulichen die Vielfalt der Forschung im Forschungsfeld BPM.

Eine neue Sicht auf BPM bietet Daniel Beverungen, der Geschäftsprozesse als organisatorische Routinen charakterisiert, die sich z. B. im Rahmen der verfügbaren IT-Artefakte entwickeln. In einer Domäne, die trotz des hohen Forschungsinteresses oftmals als limitiert in Bezug auf theoriegeführte Forschung betrachtet wird, eröffnet Beverungen somit eine interessante theoretische Perspektive. Der von ihm vorgestellte Meta-Ordnungsrahmen kombiniert organisatorische Routinen und Strukturationstheorie, um das Zusammenspiel der Gestaltung von IT, sozialen Systemen und routinebasierten Prozessen zu erklären.

Ergänzend zur routinezentrierten Sichtweise von Beverungen betrachtet die zweite Arbeit dieses Schwerpunkts Prozessflexibilität mit einem Fokus auf die Quantifizierung ihres Geschäftswerts im Dienstleistungssektor. Maximilian Röglinger, Patrick Afflerbach, Gregor Kastner und Felix Krause stellen sich der Herausforderung, das ideale $\mathrm{Maß}$ von Prozessflexibilität zu identifizieren, und nutzen dabei die Prinzipien wertorientierten Prozessmanagements. Die Anwendbarkeit des resultierenden Optimierungsmodells wird durch eine Fallstudie in der Halbleiterindustrie demonstriert.

Die dritte Arbeit von Sigrid Schefer-Wenzl und Mark Strembeck widmet sich der Verbesserung von organisatorischen Strukturen, die Geschäftsprozesse ergänzen. In prozessorientierten Informationssystemen werden Rollenkonzepte für organisatorische Entitäten genutzt und diese Arbeit erforscht, wie Delegationsrichtlinien in solchen Systemen modelliert und sichergestellt werden können. Die Empfehlungen werden mit Hilfe von UML2 und einem formalen Metamodell dargestellt.

Das Schwerpunktthema wird komplettiert durch ein Interview mit Reinhard Schütte, früheres Vorstandsmitglied, CFO und CIO bei EDEKA, zu Charakteristiken von Großprojekten und der Rolle von Prozessgestaltung und Prozessmodellierung. Im Interview beschreibt Schütte die besonderen Eigenschaften solcher "Megaprojekte" und die Herausforderungen, die er bei der Anwendung von BPM sieht, insbesondere bezüglich eines angemessenen Detaillierungsgrads der Geschäftsprozessmodelle. 
Wir danken allen Autoren, die zu diesem Schwerpunktthema beigetragen haben und im Begutachtungsprozess ein hohes Maß an Sorgfalt gezeigt haben. Ein internationales Team an Experten hat die hohe Qualität der Arbeiten sichergestellt und wir danken ihnen sehr für ihre detaillierten Kommentare. 